Revue internationale de l'économie sociale

Recma

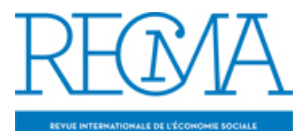

\title{
Entreprise sociale et économie sociale en Asie (première partie)
}

\section{Eric Bidet, Jacques Defourny et Marthe Nyssens}

Numéro 341, juillet 2016

Asie

Asia

URI : https://id.erudit.org/iderudit/1037434ar

DOI : https://doi.org/10.7202/1037434ar

Aller au sommaire du numéro

Éditeur(s)

Association Recma

ISSN

1626-1682 (imprimé)

2261-2599 (numérique)

Découvrir la revue

Citer ce document

Bidet, E., Defourny, J. \& Nyssens, M. (2016). Entreprise sociale et économie sociale en Asie : (première partie). Revue internationale de l'économie sociale, (341), 22-25. https://doi.org/10.7202/1037434ar d'utilisation que vous pouvez consulter en ligne. 


\title{
ENTREPRISE SOCIALE ET ÉCONOMIE SOCIALE EN ASIE (PREMIÈRE PARTIE ${ }^{(1)}$ )
}

\author{
par Eric Bidet*, Jacques Defourny** et Marthe Nyssens***
}

\footnotetext{
(1) La deuxième partie de ce dossier, qui sera consacrée à l'Asie du Sud-Est, paraîtra dans le n ${ }^{\circ} 342$ de la Recma.

(2) ICSEM est l'abréviation de «International Comparative Social Enterprise Models ». Le projet ICSEM est jalonné de réunions scientifiques au niveau régional (ICSEM Regional Symposiums) et au niveau mondial, mais aussi de ICSEM Local Talks, où les premiers résultats sont mis en débat avec les parties prenantes au niveau national. Pour plus d'informations sur le projet ICSEM: www. socent.be/icsem-project.

(3) Les ICSEM Working Papers peuvent être téléchargés à partir du site susmentionné.
}

es textes présentés dans ce dossier sont issus de travaux qui - ont été produits dans le cadre du projet ICSEM de comparaison des modèles d'entreprise sociale au niveau mondial ${ }^{(2)}$. Ce vaste projet de recherche, initié en 2013, couvre aujourd'hui plus de 50 pays à travers le monde et rassemble environ 200 chercheurs. Appuyé en premier lieu par la politique scientifique fédérale belge dans le cadre du Pôle d'attraction interuniversitaire (PAI) sur l'entreprise sociale, le projet ICSEM bénéficie aussi en France du soutien de la Fondation du Crédit coopératif et du Groupe CDC.

Avant de déboucher sur des analyses comparatives approfondies (2016-2018), la première vague des travaux ICSEM a porté sur l'identification, dans chaque pays, des principaux modèles émergents de l'entreprise sociale, sur l'analyse des contextes dans lesquels ils s'enracinent et sur les trajectoires par lesquelles la plupart d'entre eux connaissent une institutionnalisation progressive ${ }^{(3)}$.

Nous avons choisi, pour ce dossier, de mettre l'accent sur quelques-uns des pays asiatiques qui participent au projet ICSEM. Ces pays ont été répartis en deux groupes: l'un issu de l'Asie du Nord-Est, l'autre de l'Asie du Sud-Est. Les trois pays présentés dans ce premier ensemble appartiennent au bloc de l'Asie du Nord-Est; ils ont notamment en commun une culture profondément ancrée dans la philosophie confucianiste, dont les valeurs structurent encore largement la société. Si deux d'entre eux - la Corée du Sud et le Japon - sont assez proches de la France sur différents aspects, notamment en termes de niveau de développement économique et de répartition des revenus, la Chine s'en distingue très nettement et représente, de par sa taille, son niveau de développement et son système politique, un exemple d'un genre très particulier dans cet ensemble.

\footnotetext{
* Université du Mans, spécialiste de l'économie sociale et solidaire en Asie orientale.

** Université de Liège, directeur du Centre d'économie sociale à HEC-Liège.

*** Université catholique de Louvain, présidente du Cirtes.

M. Nyssens et J. Defourny assurent ensemble la coordination scientifique du projet ICSEM.
} 
Quelques indicateurs clés sur les pays représentés

\begin{tabular}{|l|l|l|l|l|l|}
\hline & France & Chine & Corée & Japon & Unité (année) \\
\hline Population & 67 & 1367 & 49 & 127 & millions (2015) \\
\hline PIB-PPA/hab. & 35700 & 9800 & 33200 & 37100 & millions \$ (2013) \\
\hline Taux croissance & 0,7 & 7,7 & 2,9 & 1,6 & $\%(2013)$ \\
\hline IDH & $0,888(22)$ & $0,727(90)$ & $0,898(17)$ & $0,891(20)$ & $(2014)$ \\
\hline Gini 2013 & 0,331 & 0,473 & 0,302 & n.d. & \\
\hline Gini 2010 & 0,30 & 0,41 & 0,31 & 0,34 & \\
\hline Dépenses santé/hab. & 4864 & 367 & 1703 & 3966 & $\$(2013)$ \\
\hline Taux chômage & 10,8 & 4,1 & 5,7 & 2,9 & $\%(2014)$ \\
\hline
\end{tabular}

Ces trois pays nord-asiatiques sont marqués par une tradition coopérative et associative dont les racines sont très anciennes, mais qui est longtemps restée sous le strict contrôle de l'administration et du pouvoir politique, qu'il ait été d'idéologie communiste en Chine ou anti-communiste au Japon, et plus encore en Corée. Ce n'est qu'assez récemment que cette tutelle publique s'est estompée au Japon ou en Corée, qu'elle s'est atténuée en Chine et que des organisations coopératives et associatives autonomes ont pu émerger et se développer, jusqu'à être progressivement reconnues au plan juridique. Ces pays ont en effet cherché dans des nouvelles formes de partenariat public-privé une réponse à des problématiques sociales communes, souvent à la frontière des politiques d'emploi et des politiques sociales, ou en lien avec les questions de territoire, de développement communautaire ou d'environnement. C'est dans cette perspective qu'a émergé un intérêt nouveau pour les entreprises sociales, les associations ou de nouvelles formes de coopératives, telles que les coopératives de consommation, les coopératives médicales, les coopératives rurales ou les coopératives sociales.

A cet égard, la Corée est sans aucun doute le pays le plus avancé des trois, avec la mise en place de plusieurs dispositifs destinés à promouvoir l'entreprise sociale, qui a été identifiée dès la fin des années 1990 comme un outil important en matière de politique d'emploi. Après l'introduction d'une loi spécifique de promotion de l'entreprise sociale en 2006, différents dispositifs complémentaires, régionaux ou sectoriels, sont apparus. L'article sur la Corée souligne que l'un des effets de cette dynamique a été notamment de générer un intérêt pour le modèle coopératif, ce qui s'est notamment traduit en 2012 par l'adoption d'une loi coopérative générale offrant une plus grande reconnaissance et de nouvelles perspectives au modèle coopératif. Dans le sillage de l'intérêt pour l'entreprise sociale a également émergé une réflexion sur l'économie sociale, qui pourrait déboucher assez rapidement sur l'adoption d'une loi-cadre. 
A l'inverse de l'expérience française, c'est donc à partir d'un intérêt initial pour l'entreprise sociale qu'émerge en Corée une véritable reconnaissance du modèle coopératif et que se construit une définition de l'économie sociale, qui était jusqu'à présent embryonnaire et idéologiquement mal perçue dans ce pays fortement marqué par la partition Nord-Sud.

Comme la Corée, le Japon a un mouvement coopératif très dynamique, initialement basé sur les activités agricoles et bancaires, et étroitement lié au secteur public, qui présente toutefois la particularité d'avoir intégré plus rapidement le modèle plus indépendant de la coopérative de consommateurs. A partir des années 1970, à côté de ces acteurs traditionnels du mouvement coopératif, sont apparues de nouvelles formes de coopératives, initiées par des groupes d'agriculteurs ou de citoyens pour répondre à des problématiques territoriales, telles que la préservation des terres agricoles ou la promotion d'une agriculture biologique. Ces « coopératives communautaires » se sont le plus souvent développées en partenariat avec les autorités locales. Elles représentent à la fois un renouvellement du modèle coopératif traditionnel et incarnent, dans le contexte japonais, une des formes que prend l'entreprise sociale basée sur le modèle coopératif.

Le cas de la Chine diffère nettement des deux premiers en raison d'une transition beaucoup plus récente vers un modèle d'économie de marché, ce qui se traduit à la fois par un niveau de développement très différent dans les trois registres de l'indice de développement humain (IDH) et vis-à-vis des indicateurs relatifs à la protection sociale, la pauvreté ou les inégalités. Dans ce contexte, malgré l'absence de statut juridique spécifique, sont apparues toutes sortes d'associations et d'entreprises sociales pour satisfaire des besoins sociaux auxquels ni l'Etat ni le marché n’apportent une réponse suffisante. Beaucoup restent cependant étroitement contrôlées par le gouvernement montrant, comme en Corée ou au Japon, que les questions d'autonomie et de gouvernance constituent sans doute, dans ces pays asiatiques, un point de divergence important avec les organisations occidentales.

A travers ces trois contributions nationales, on perçoit le remarquable dynamisme qui existe en Asie du Nord-Est en matière d'économie sociale et d'entreprise sociale. Par leur singularité, ces expériences apportent un éclairage nouveau sur la façon dont ces deux concepts très proches peuvent s'articuler. Nous avançons l'hypothèse qu'entreprise sociale et économie sociale, au lieu de principalement se concurrencer, tendent plutôt à se renforcer mutuellement: la montée de l'entreprise sociale fournit une opportunité et un véhicule paré des atouts de la nouveauté, qui lui-même permet de revisiter et de redécouvrir des réalités d'économie sociale, surtout coopératives et de plus 
en plus aussi associatives. A leur tour, bien des expériences coopératives, à la fois novatrices et souvent adossées à des mouvements plus anciens, fournissent des gages de maturité et de crédibilité à des nébuleuses d'entreprises sociales qui doivent encore largement faire leurs preuves.

C'est là l'une des pistes que nous poursuivrons avec trois autres contributions issues du projet ICSEM, d'Asie du Sud-Est cette fois, qui seront publiées dans le prochain numéro de la Recma. 